\title{
Frontal Cystic Meningioma Removed by a Partial Transfrontal Craniotomy in a Cat
}

\author{
Kihoon Kim
}

\begin{abstract}
Background: Meningiomas are the most frequently reported intracranial tumors in cats. It is known to arise at the point of arachnoid cells project into the dural venous sinuses. Cats with intracranial meningiomas are treated by surgical management as the tumors are commonly delineated from normal brain tissue and are not likely to adhere to the cerebral parenchyma. Although meningioma is the most common intracranial tumor in cats, the incidence of cystic meningioma is low. The objective of the current study is to report a case of frontal meningioma with peritumoral cystic structure removed by a partial transfrontal craniotomy.

Case: A 10-year-old castrated British shorthair cat was referred to the Baeksan Feline Medical Center with a recent onset of seizures. On the physical examination, the patient was bright and alert. Neurological examinations were unremarkable at the time of presentation. Hematologic examinations were within normal limits. Thoracic and abdominal radiography, and abdominal ultrasonography revealed unremarkable findings. Magnetic resonance imaging revealed an extra-axial mass cranial to the frontal lobe. On the sagittal plane, a cystic structure was identified in the frontal area on post-contrast T1W images. No contrast enhancement of the cystic wall was identified after intravenous injection of contrast medium on T1W. On the transverse plane of T2W images, midline shift to the left due to peritumoral edema was observed. The mass was removed via partial transfrontal craniotomy. Postoperative radiography was performed to ensure appropriate placement of the mesh. The patient recovered uneventfully after anesthesia. After the surgery, the patient was closely monitored in an intensive care unit between 24 and $48 \mathrm{~h}$. Based on the histologic findings, the final diagnosis was a fibroblastic meningioma. Nineteen months after the surgery, there was no seizure activity identified by the owner.

Discussion: Depending on the location of the cyst, meningiomas can be classified into 4 types according to the human literature. In types 1 and 2, the whole cyst is located within the tumor, resulting in contrast enhancement of the cystic wall. In types 3 and 4, the cysts are located outside the tumor, and no contrast enhancement of the cystic wall is observed. In type 3, the cyst lies adjacent to the brain parenchyma rather than adjacent to the tumor and the meningioma is related to a cerebrospinal fluid cyst bordered by the arachnoid. It is important to classify the type of cystic meningioma prior to surgery in order to decide whether to remove the cystic wall. In type 2, the cystic wall is infiltrated by tumor cells, while the cystic wall of type 3 meningioma is composed of gliotic tissue without any tumor cells. Therefore, in type 2, the meningiomas with cystic walls should be removed for the prevention of recurrence, while in type 3 meningioma, the tumor can be managed by cyst decompression and excision of the solid component. Based on the Nauta classification, the cystic meningioma reported here was considered to be type 3 . Therefore, the surgical procedure aimed to remove the solid component of the mass, leaving the cystic wall attached to the normal brain. As the solid part of the meningioma was located beneath the internal plate of the left frontal bone, the partial transfrontal craniotomy was sufficient to expose and remove the entire mass. To the author's knowledge, this is first case report describing a patient with frontal meningioma with a peritumoral cyst removed by a partial transfrontal craniotomy based on the Nauta classification.
\end{abstract}

Keywords: cat, meningioma, peritumoral cyst, partial transfrontal craniotomy.

DOI: $10.22456 / 1679-9216.112133$ 


\section{INTRODUCTION}

Meningiomas are the most common intracranial tumors in cats with no breed predisposition [1,11], having an occurrence of $2.2 \%$ according to a previous study [10]. The tumor may be single or multiple and seems to have a slow growth [12]. It is known that meningiomas arising at the point of arachnoid cells project into the dural venous sinuses [5,9]. In cats, the supratentorial meninges, the tela choroidea of the third ventricle, and the cerebellar meninges are the most common locations of meningioma [7]. Although meningioma is the most frequently reported intracranial tumor in cats, cystic meningioma is rarely reported.

Intracranial meningiomas in humans are removed surgically, and recurring tumors are treated with radiotherapy or subsequent surgical removal [7]. In contrast, cats with intracranial meningiomas are treated by surgical management as the tumors are commonly delineated from normal brain tissue and are not likely to infiltrate the cerebral parenchyma $[1,7]$. Herein, this report describes the frontal meningioma with peritumoral cyst in a cat removed successfully by a partial transfrontal craniotomy.

\section{CASE}

A 10-year-old castrated British shorthair cat was referred to the Baeksan Feline Medical Center for a recent onset of seizure. On the physical examination, the patient was bright, alert, and adequately hydrated. Neurological examinations were unremarkable at the time of presentation. Hematologic examinations, such as complete blood count, serum biochemical profile, electrolyte analysis, and FeLV/FIV, were normal. Thoracic and abdominal radiography, and abdominal ultrasonography revealed unremarkable findings.

For further neurologic examination, magnetic resonance imaging ${ }^{1}$ (1.5T MRI) was performed and $\mathrm{T}-1$ weighted (T1W) and T-2 weighted images (T2W) were acquired. MRI revealed an extra-axial mass cranial to the frontal lobe. On the sagittal plane, a cystic structure was identified in the frontal area on post-contrast $\mathrm{T} 1 \mathrm{~W}$ images (Figure 1A) and hyperintense on T2W images (Figure 1B); compression of the cerebellum to the caudodorsal side due to the tentorial herniation was also identified (Figure 1C). On the dorsal plane, the dural tail sign extended along the cerebral meninges (Figure 1D), and no contrast enhancement of the cystic wall (Figure 1E) was observed after intravenous injection of contrast medium2 [Magnevist ${ }^{\circledR}-0.2 \mathrm{~mL} / \mathrm{kg}$, i.v.] on $\mathrm{T} 1 \mathrm{~W}$. On the transverse plane of $\mathrm{T} 2 \mathrm{~W}$ images, midline shift to the left due to peritumoral edema was observed, and the mass was identified (Figure 1F).

Before surgery, the patient received butorphanol3 [Butopahn ${ }^{\circledR}-0.5 \mathrm{mg} / \mathrm{kg}$, i.v.] and cefotaxime4 [30 $\mathrm{mg} / \mathrm{kg}$, i.v.] prior to induction with alfaxalone5 [Alfaxan ${ }^{\circledR}-4 \mathrm{mg} / \mathrm{kg}$, i.v.]. Mannitol6 [D-Mannitol® $-0.5 \mathrm{~g} / \mathrm{kg}$, slow i.v.) was also administered at the time of induction for reduction of the intracranial pressure. General anesthesia was maintained with isoflurane7 [Isoflurane $\left.{ }^{\circledR}\right]$ with oxygen after intubation; $0.9 \%$ normal saline 6 [constant rate infusion of $5 \mathrm{~mL} / \mathrm{kg} / \mathrm{h}$ ], furosemide8 [Lasix ${ }^{\circledR}-2 \mathrm{mg} / \mathrm{kg}$, i.v.], and dexamethasone9 [2 mg/kg, i.v.] were administered intraoperatively.

The patient was positioned in sternal recumbency, with its head slightly elevated. A skin incision was made along the dorsal midline of the frontal bone (Figure 2A). Craniotomy on the right frontal bone was performed with a nitrogen-powered drill10 with a round carbide burr. The craniotomy site was then extended using rongeur forceps. As the mass was located in the frontal region, a partial transfrontal craniotomy was applied to achieve additional exposure of the mass (Figure 2B). After drainage of the cystic fluid with a $24 \mathrm{G}$ syringe, the entire mass was removed by gentle dissection from the surrounding frontal lobe. To cover the skull defect, a titanium wire mesh and screws 10 were applied (Figure 2C). Postoperative radiography was performed to ensure appropriate placement of the mesh (Figure 2D). The patient recovered uneventfully after anesthesia. After the surgery, the patient was closely monitored in an intensive care unit between 24 and $48 \mathrm{~h}$. For pain management, fentanyl11 [Fentanyl citrate inj. ${ }^{\circledR}$ was constantly administrated i.v. to the patient at a rate of $3 \mu \mathrm{g} / \mathrm{kg} / \mathrm{h}$ during the first $24 \mathrm{~h}$ ], and a fentanyl patch 12 [Durogesic ${ }^{\circledR}-12 \mu \mathrm{g} / \mathrm{h}$ ] was then applied to the left abdomen. Postoperative complications including epistaxis and knuckling of the left forelimb were observed, which were resolved in 3 and 5 days, respectively. The patient was discharged on day 7 and revealed normal gait at day 14, when the sutures were removed. Follow-up assessment was performed via telephone reports from the owner 19 months after surgery and the owner reported the patient was normal without any neurological sign.

The removed mass was stationary and firm (Figure 3A), and was routinely processed and stained 
with hematoxylin and eosin for histological evaluation. It was composed of interlacing bundles and streams of spindle cells within a typically thick, collagenous stroma. Neoplastic cells had mild anisocytosis and anisokaryosis, as well as one mitotic figure counted in 10 high-powered fields. Vascular invasion was not identified. These findings were all consistent with spindle cell neoplasm, which is most likely fibroblastic subtype of meningioma (Figure 3B).

\section{DISCUSSION}

Depending on the location of the cyst, meningiomas can be classified into 4 groups according to the human literature [8] . In types 1 and 2, the whole cyst is located within the tumor, resulting in contrast enhancement of the cystic wall. In type 1, the cyst is centrally located within the tumor, while in type 2 , some part of the cyst is identifiable on the wall of the tumors, and the remaining part of the cyst wall consists of the tumor cells. In types 3 and 4, the cysts are located outside the tumor, and no contrast enhancement of the cystic wall is observed. In type 3 , the cyst lies adjacent to the brain parenchyma rather than adjacent to the tumor and the meningioma is related to a cerebrospinal fluid cyst bordered by the arachnoid. It is important to classify the type of cystic meningioma before surgery to decide whether to remove the cystic wall [13]. In type 2 , the cystic wall is infiltrated by tumor cells, while the cystic wall of type 3 meningioma is composed of gliotic tissue without any tumor cells. Therefore, in type 2 , the meningiomas with cystic walls should be removed for the prevention of recurrence, while in type 3 meningioma, the tumor can be managed by cyst decompression and excision of the solid component. However, according to Ferrante et al. [2], types 2 and 3 are not always enhanced by contrast medium, indicating that the Nauta classification is not sufficient to differentiate between the two types.

In contrast, there are several hypotheses regarding the correlation between the mechanism of fluid formation and the type of cystic meningioma [13]. In types 1 and 2, the cystic fluid originates from the secretion of functional tumor cells, whereas, in type 3, the fluid originates from the evolution of peritumoral edema into a peritumoral cyst. In this mechanism, the cerebrospinal fluid accumulates near the tumor by decreased resorption. Considering the peritumoral edematous changes adjacent to the cystic structure, in

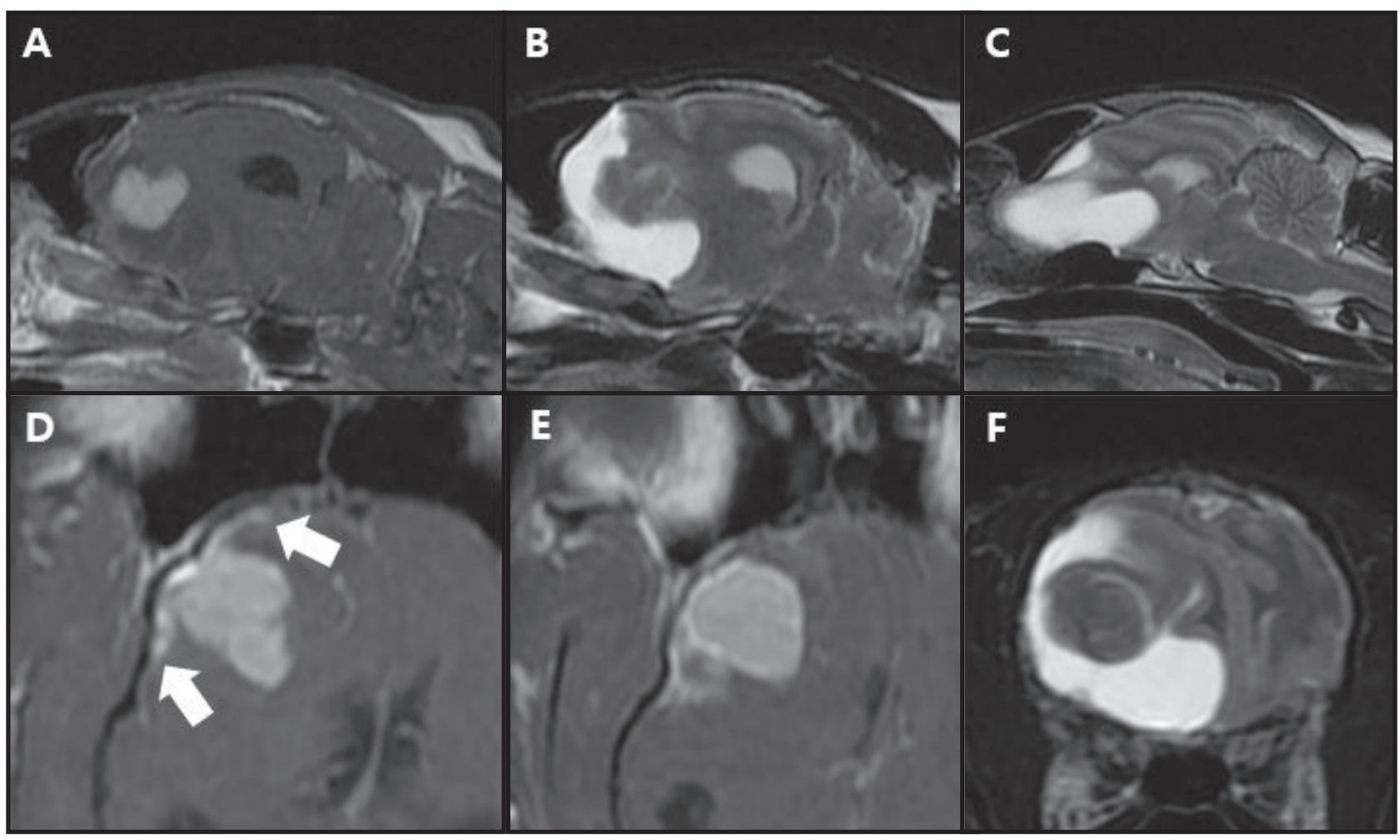

Figure 1. Magnetic resonance imaging scans showing a frontal mass with cystic structure. A- Frontal hypointense mass on a post-contrast T1-weighted (T1W) image. B- Frontal hyperintense mass on a T2-weighted (T2W) image. C- Compression of the cerebellum to the caudodorsal side due to the tentorial herniation. D- Dural tail sign (arrow) extending from the cerebral meninges on a dorsal post-contrast T1W image. E- No contrast enhancement of the cystic wall was observed after insertion of contrast medium on a T1W image. F- Deviation of midline shift to the left by peritumoral cyst and the mass identified on the transverse plane of a T2W image. 


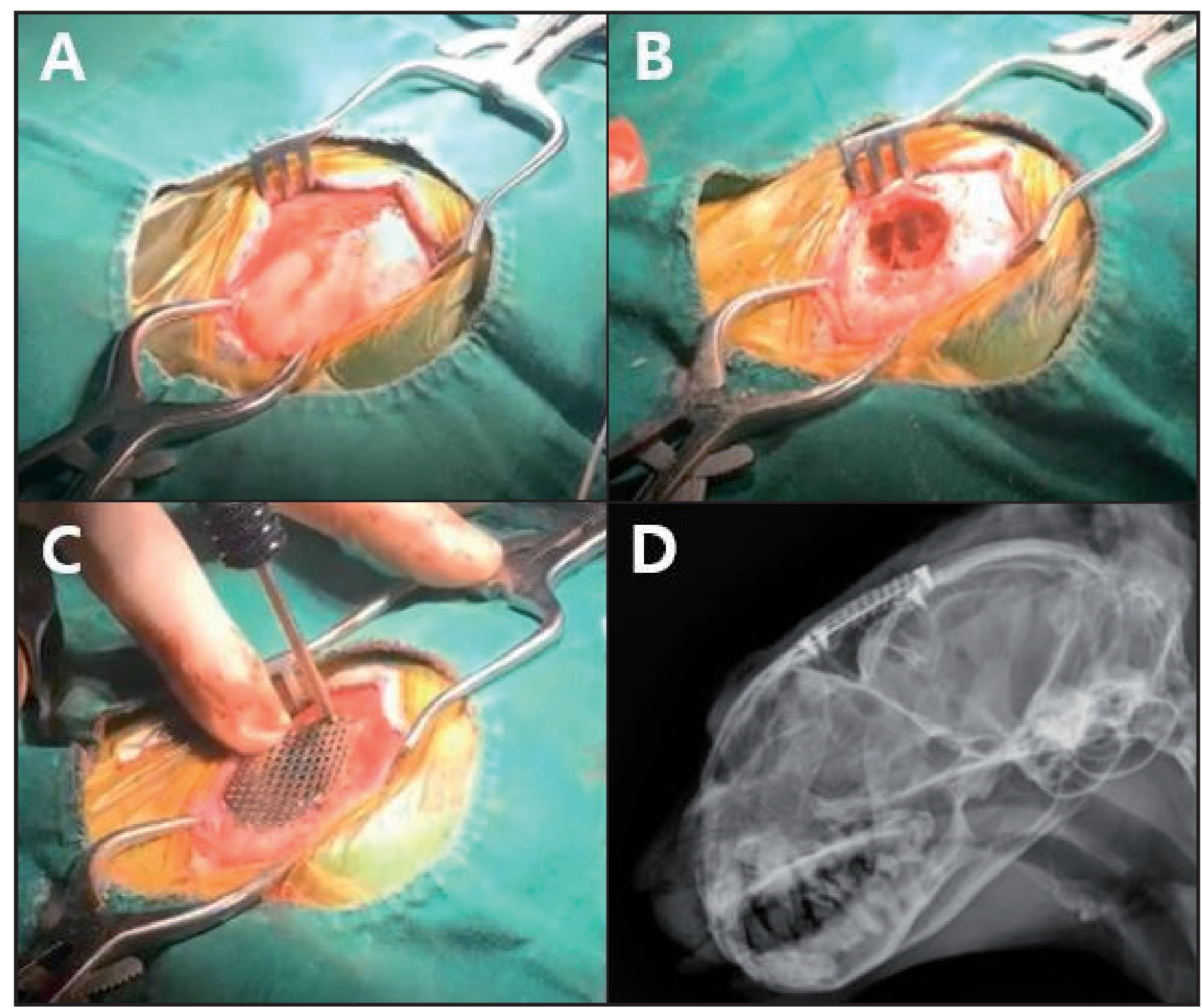

Figure 2. Surgical procedure and postoperative radiography. A- The subcutaneous tissues and frontal muscles were reflected laterally on each side of the incision. B- The cystic structure was exposed after removal of the internal plate of the frontal bone. C- Titanium wire mesh covering skull defect. D- Postoperative radiographic image.

the case reported here, it is evident that the cystic meningioma belongs to type 3. Based on the Nauta et al. [8] classification and peritumoral edematous changes, the cystic meningioma reported here was considered to be type 3 .

Several approaches for the removal of meningioma by craniotomy in companion animals have been previously reported $[3,4,11]$. The surgical approach is determined depending on the location of the meningioma. A rostrotentorial craniectomy is a commonly utilized technique to expose the majority of the mass in cats [4]. For frontal meningioma in cats, additional exposure techniques, such as the partial transfrontal approach, can be combined with rostrotentorial craniectomy. In this case, a tentative diagnosis of type 3 cystic meningioma was made before surgery, and the surgical procedure aimed to remove the solid component of the mass and leave the cystic wall attached to the normal brain. As the solid part of the meningioma was located beneath the internal plate of the left frontal bone, the partial transfrontal craniotomy which is less invasive compared to rostrotentorial craniectomy was sufficient to expose and remove the mass.

Although meningiomas are frequently reported in cats previously, there is only one case report describing pericystic meningioma in cat. To the author's knowledge, this is first case report describing a patient with frontal meningioma with a peritumoral cyst removed by partial transfrontal craniotomy. Although the Nauta classification based on human meningioma was used for the determination of the surgical plan without considering the cystic wall component, the patient was 


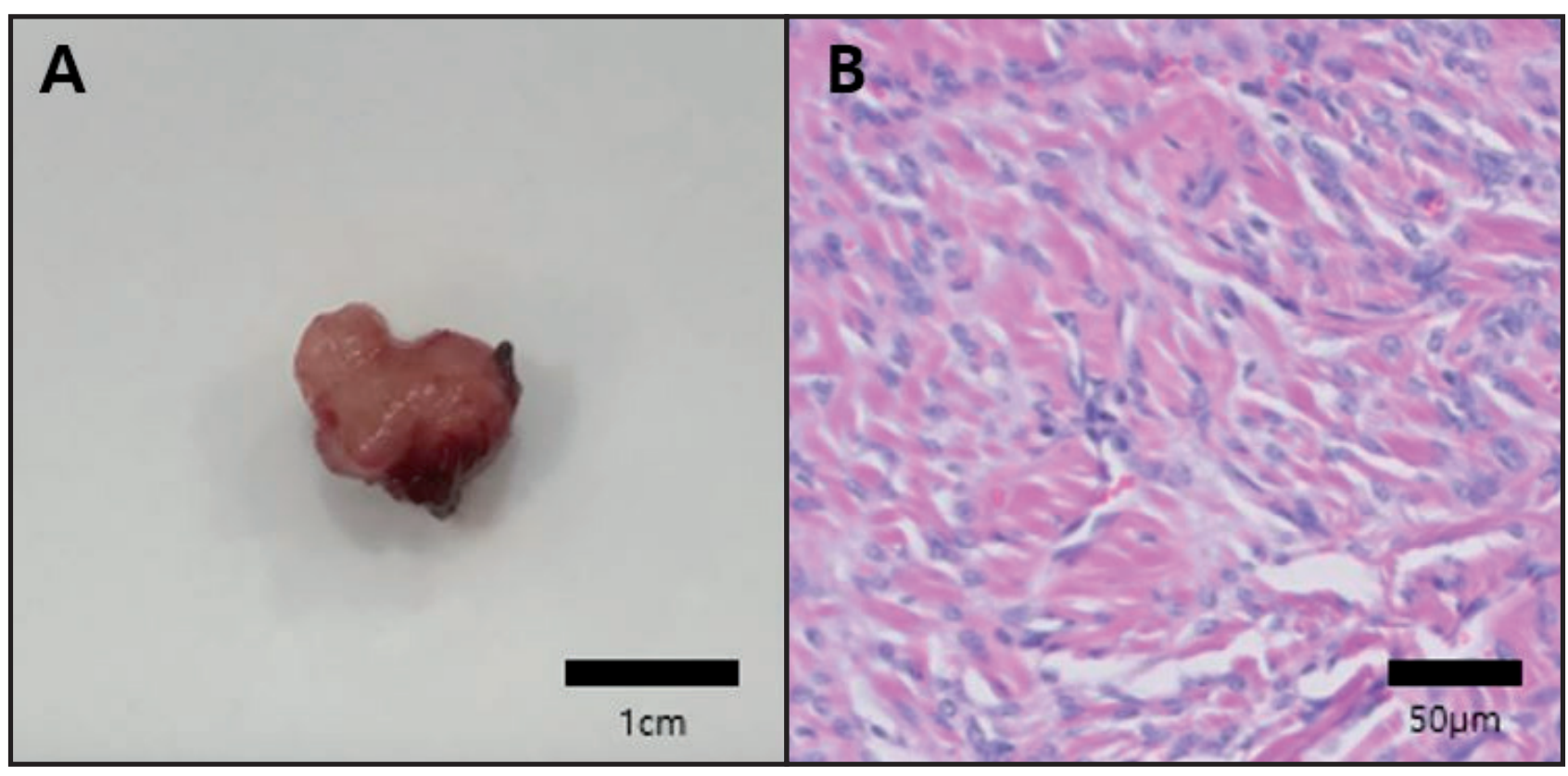

Figure 3. A- Gross appearance of the mass. B- The dominant component of the tumor was the bundles of spindle-shaped cells, which were arranged in a collagenous stroma $[\mathrm{HE} ; \times 400]$.

reported as normal via telephone interview with the owner 19 months after surgery. However, more cases of feline cystic meningioma are required to establish the classification in cats.

\section{MANUFACTURERS}

${ }^{1}$ GE healthcare. Milwaukee, WI, USA.

${ }^{2}$ Beyer-Schering Pharmaceutical. Berlin, Germany.

${ }^{3}$ Myungmoon Pharmaceutical. Seoul, Korea.

${ }^{4}$ Chongkundang. Seoul, Korea.

${ }^{5}$ Jurox. Rutherford, Australia.
${ }^{6}$ Daehan Pharmaceutical. Seoul, Korea.

${ }^{7}$ Choongwae Pharmaceutical. Seoul, Korea.

${ }^{8}$ Dongwha Pharmaceutical. Seoul, Korea.

${ }^{9}$ Jeil Pharmaceutical. Seoul, Korea.

${ }^{10} \mathrm{i}$-Medicom. Seoul, Korea.

${ }^{11}$ Hana Pharmaceutical. Seoul, Korea.

${ }^{12}$ Jannsen Korea. Seoul, Korea.

Declaration of interest. The author reports no conflicts of interest. The author alone is responsible for the content and writing of the paper.

\section{REFERENCES}

1 Adamo P.F., Forrest L. \& Dubielzig R. 2004. Canine and feline meningiomas: Diagnosis, treatment, and prognosis. Compendium on Continuing Education for the Practising Veterinarian. 26: 951-965.

2 Ferrante L., Acqui M., Lunardi P., Qasho R. \& Fortuna A. 1997. MRI in the diagnosis of cystic meningiomas: Surgical implication. Acta Neurochirurgica. 139: 8-11

3 Glass E., Kapatki, A., Vite C. \& Steinberg S. 2014. A modified bilateral transfrontal sinus approach to the canine frontal lobe and olfactory bulb: surgical technique and five cases. Journal of the American Animal Hospital Association. 36(1): 43-50.

4 Gordon L.E., Thacher C., Matthiesen D.T. \& Joseph R.J. 1994. Results of craniotomy for the treatment of cerebral meningioma in 42 cats. Veterinary Surgery. 23(2): 94-100.

5 Kitagawa M., Kanayama K. \& Sakai T. 2002. Cystic meningioma in a dog. Journal of Small Animal Practice. 43(6): 272-274.

6 Lawson D., Burk R.L. \& Prata R.G. 1984. Cerebral meningioma in the cat: diagnosis and surgical treatment of ten cases. Journal of the American Animal Hospital Association. 20: 333-342.

7 Motta L., Mandara M.T. \& Skerritt G.C. 2012. Canine and feline intracranial meningiomas: An updated review. Veterinary Journal. 192(2): 15-165.

8 Nauta H., Tucker W., Horsey W., Bilbao J. \& Gonsalves C. 1979. Xanthochromic cysts associated with meningioma. Journal of Neurology, Neurosurgery and Psychiatry. 42(6): 529-535. 
9 Summer B.A., Cummings J. \& DeLahunta A. 1995. Tumours of the central nervous system. In: Summers B.A., Cummings J.F. \& DeLahunta A. (Eds). Veterinary Neuropathology. St. Louis: Mosby, pp.351-401.

10 Troxel M.T., Vite C.H., Massicotte C., Mclear R.C., Van Winkle T.J., Glass E.N., Teeches D. \& Dayrell-Hart B. 2004. Magnetic resonance imaging features of feline intracranial neoplasia: Retrospective analysis of 46 cats. Journal of Veterinary Internal Medicine. 18: 176-189.

11 Volckaert V. \& Royaux E. 2020. Peritumoral (pseudo) cystic meningioma in a cat. Vlaams Diergeneeskdig Tijdschrift. 89(4): 215-220.

12 Wills T.B., Chen A.V.\& Haldorson G.J. 2009. What is your diagnosis? Intracranial mass in a cat. Veterinary Clinical Pathology. 38(1): 39-41.

13 Zee C.S., Chen T., Hinton D.R., Tan M., Segall H.D. \& Apuzzo M.J. 1995. Magnetic resonance imaging of cystic meningiomas and its surgical implications. Neurosurgery. 36(3): 482-488. 